\title{
Computational identification of six salt stress responsive genes in Cynara scolymus $\mathbf{L}$.
}

\author{
Iftekhar Ahmed Baloch ${ }^{1 *}$, Saima Johar ${ }^{2}$, Bibi Hani Mengal ${ }^{2}$, Ambreen \\ Khadija Alvi ${ }^{3}$, Shehnaz Shair Qambrani ${ }^{4}$ Muhammad Naeem Shawani ${ }^{4}$ \\ and Bibi Sadia ${ }^{2}$ \\ 1. Department of Botany, Govt. Postgraduate Science College Quetta-Pakistan \\ 2. Department of Plant Sciences, Sardar Bahadur Khan Women's University Quetta-Pakistan \\ 3. Department of Botany, Govt. College Women University Faisalabad-Pakistan \\ 4. Department of Biotechnology and Informatics, Balochistan University of Information Technology, Engineering \\ and Management Sciences (BUITEMS) Quetta-Pakistan \\ *Corresponding author's email: iftekharbaloch@gmail.com \\ Citation \\ Iftekhar Ahmed Baloch, Saima Johar, Bibi Hani Mengal, Ambreen Khadija Alvi, Shehnaz Shair Qambrani, \\ Muhammad Naeem Shawani and Bibi Sadia. Computational identification of salt stress responsive genes in Cynara \\ Scolymus L. Pure and Applied Biology. Vol. 9, Issue 3, pp1723-1736. http://dx.doi.org/10.19045/bspab.2020.90183

\begin{tabular}{llll}
\hline \hline Received: 05/04/2019 & Revised: 03/04/2020 & Accepted: 08/04/2020 & Online First: 09/04/2020 \\
\hline
\end{tabular}

\section{Abstract}

Cynara scolymus L. is a perennial plant belonging to family Asteracea. It has many traditional and medicinal values and is also used as a source of vegetable in many countries. This plant thrives well in saline areas and is considered to be moderately salt tolerant but very little information is available about its salt stress tolerance mechanism. In this study six (6) salt stress responsive genes (Four up-regulated i.e. CSD1, RCI3, AFB3, HVA22A and two down-regulated i.e. CSD2, MSD1) were identified in Cynara scolymus L. by using homology search approach with the help of various bioinformatics tools/resources (BLASTn, BLASTp, ORF finder, CD search, Clustal Omega, ProtParam and Phyre2). Furthermore, the secondary and tertiary structures of reference and target genes were predicted and compared for confidence and similarities in structure. All selected salt stress responsive genes were predicted in Cynara scolymus through computational analysis of 36,323 ESTs. The EST based identification of these genes is a confirmation of their expression and these findings will help us understand the salt stress tolerance mechanism in this important plant.

Keywords: Bioinformatics; Cynara scolymus; Salt stress genes

\section{Introduction}

Cynara scolymus L. (C. scolymus) is a perennial plant commonly known as Globe Artichoke and belongs to family Asteraceae. It is a rich source of minerals, fiber and phenolics [1, 2]. Its leaf extract is used as antimicrobial, hepatoprotective and for cholesterol reducing purposes. The eatable parts of $C$. scolymus are the delicate internal leaves and the receptacle called as "heart" [3]. It assumes an essential part in humans' daily nutritional value, mainly in the Mediterranean regions [4]. C. scolymus has a small amount of fat and large amount of minerals like potassium $(\mathrm{K})$, sodium $(\mathrm{Na})$, phosphorus $(\mathrm{P})$, vitamin $\mathrm{C}$, polyphenols and 
flavones [3, 5]. Many other Cynara species are grown in different areas of the world i.e. in U.S, principally in California, in South America, North Africa, Near East and China $[4,5]$.

The condition in which plants grow in higher than normal quantity of salt is called salinity or salt stress condition. Salt stress is an important abiotic stress which affects the metabolic and physiological responses of the plants. It is one of the main abiotic stresses faced by plants. Plants identify the stress signals and pass them to the cellular apparatus and then the adaptation is fulfilled by the regulation of gene expression [6]. $C$. scolymus L. grows in high salinity and drought conditions [7]. Since it is a medicinal plant, researchers have also studied the effect of salinity on its phenolic composition and antioxidant activity [8].

Salt stress genes have been extensively reported in model plants i.e. Oryza sativa and Arabidopsis thaliana. Since many salt tolerant plants express their salt tolerance genes, here we hypothesize that such salt stress responsive genes may also be present in Cynara scolymus. This research is an attempt to identify and annotate some salt stress responsive genes (Four up-regulated i.e. CSD1, RCI3, AFB3, HVA22A and two down-regulated i.e. CSD2, MSD1) in $C$. scolymus by using different bioinformatics tools (BLASTn, BLASTp, ORF finder, CD search, Clustal Omega, ProtParam and
Phyre2). A series of steps were performed to find the salt stress responsive genes in $C$. scolymus.

\section{Materials and methods}

A total of six (6) salt stress responsive genes (CSD1, RCI3, AFB3, HVA22A, CSD2 and MSD1) were selected from the model plant Arabidopsis thaliana through literature survey [9-12]. An attempt was made to identify the potential orthologs of these reference genes in $C$. scolymus. The main steps of methodology are discussed below.

Retreival of selected gene sequences and their Open Reading Frames (ORFs).

Messenger RNA (mRNA) sequences of salt stress genes of the Arabidopsis thaliana were fetched by using gene database publically available at National Center for Biotechnology Information (NCBI) and saved in FASTA format for downstream analysis. FASTA sequence of each gene was subjected to ORF finder tool publically available at NCBI. Longest open reading frames of each gene were saved. The nucleotide and protein sequences of these genes were used to find their potential orthologs in Cynara scolymus. Gene symbol, Accession No of Reference Genes (from Arabidopsis thaliana), Longest ORF of Reference Gene (from Arabidopsis thaliana) and Accession No of target Genes (predicted in C. scolymus) are shown in (Table 1).

Table 1. Gene symbol, Accession No and ORFs of Reference (Arabidopsis thaliana) and accession no of target Genes (predicted in Cynara scolymus).

\begin{tabular}{|c|c|c|c|}
\hline $\begin{array}{c}\text { Gene } \\
\text { symbol }\end{array}$ & $\begin{array}{c}\text { Accession No of Reference Gene } \\
\text { (Arabidopsis thaliana) }\end{array}$ & $\begin{array}{c}\text { Longest ORF of Reference } \\
\text { Gene } \\
\text { (Arabidopsis thaliana) }\end{array}$ & $\begin{array}{c}\text { Accession No of } \\
\text { target EST } \\
\text { (Cynara scolymus) }\end{array}$ \\
\hline CSD1 & NM_100757.4 & +3 & GE599954.1 \\
\hline RCI3 & NM_100405.4 & +1 & GE578194.1 \\
\hline AFB3 & NM_101152.5 & +1 & GE583634.1 \\
\hline HVA22A & NM_118628.4 & +2 & GE589804.1 \\
\hline CSD2 & NM_128379.4 & +2 & GE599319.1 \\
\hline MSD1 & NM_111929.4 & +1 & GE594969.1 \\
\hline
\end{tabular}




\section{Homology search}

Basic Local Alignment Search Tool (BLAST) is an important tool for ortholog search. The available 36,323 Expressed Sequence Tags (ESTs) of Cynara scolymus were computationally screened to search the selected genes. The nucleotide ORF sequences of Arabidopsis thaliana genes were subjected to nucleotide Blast (BLASTn) against ESTs of Cynara scolymus and amino acid sequences of ORFs were subjected to protein Blast (BLASTp). On the basis of query coverage and percentage identity the best matched ESTs and protein sequences were selected and their sequences were saved in FASTA format.

\section{Finding conserved domains}

Best matched ESTs of Cynara scolymus and genes of the model plant Arabidopsis thaliana were subjected to Conserved Domain Database (CD Search) to detect the presence and position of conserved domains which perform functions in saline conditions. Conserved domains of the Arabidopsis thaliana genes were compared with the conserved domains of best matched ESTs of Cynara scolymus by this method.

Phylogenetic analysis and Percent Identity Matrix of MSD1 gene

Freely available Multiple sequence alignment (MSA) tool, Clustal Omega was used for phylogenetic analysis of MSD1 gene. A Cladogram was constructed for MSD1 due to its conserved nature in many plants. Amino acid sequences of newly predicted MSD1 gene in C. scolymus was compared with that of Camelina sativa, Papaver somniferum and Arabidopsis thaliana. Percent Identity Matrix was also calculated for each MSD1 gene sequence. The results were saved.

\section{Deduction of secondary and tertiary protein structures}

The physical properties including Theoretical PI, Instability Index, Aliphatic Index and
GRAVY of the deduced protein structures of the target $C$. scolymas ESTs and Arabidopsis thaliana reference genes were obtained using online tool ProtParam from the ExPasy server [13]. Whereas, the secondary and tertiary structures were obtained from Phyre2 online protein analysis server [14]. The deduced secondary and tertiary structures were compared for confidence and similarities in structure.

\section{Results}

Identification of new conserved salt stress genes in $C$. scolymus

All selected salt stress responsive genes were identified in Cynara scolymus through computational analysis of 36,323 ESTs. Homology search of each gene showed less query coverage and identity when compared with nucleotides (blastn) and comparatively higher query coverage and identity when compared with protein sequences (blastp). Query coverage of nucleotide and protein sequences of CSD1, RCI3, AFB3, HVA22A, CSD2 and MSD1 are shown in (Fig.1a \& b). Identification of similar conserved domains in predicted genes

CD search results of newly predicted conserved genes in Cynara scolymus demonstrated that all predicted genes share almost similar conserved domains with their corresponding reference genes. Similarities of conserved domains are shown in (Fig. 2a to f).

Phylogenetic analysis and Percent Identity Matrix of MSD1 genes

Phylogenetic analysis of the protein coding sequence (amino acid) of MSD1 gene with its orthologs in Camelina sativa, Papaver somniferum and Arabidopsis thaliana showed that MSD1 gene of Cynara scolymus is more close to Papaver somniferum as compred to Camelina sativa and Arabidopsis thaliana (Fig. 3a \& b). Percent Identity Matrix (as predited by Clustal Omega) for 
MSD1 gene of each plant is shown in (Table 2).

\section{Deduction of secondary and tertiary structures of proteins}

In current analysis the deduced secondary and tertiary structures of proteins also showed similar physical properties including molecular stability, aliphatic index and GRAVY.

The results in (Table 3) showed that the $C$. scolymus target ESTs coded for proteins showed homology to the respective Arabidopsis reference genes. All deduced proteins of target ESTs except GE578194.1 showed similar stability / instability to their respective Arabidopsis reference counterparts. Values of aliphatic indices deviated within the range of +-11 to the reference protein values which indicated comparable contribution of the aliphatic side chains to the stability of the protein molecules. All target EST deduced proteins showed comparable polarity to the reference sequence proteins except for the EST sequence GE583634.1, which gave a very low positive GRAVY value.

The deduced secondary and tertiary structures of target ESTs selected from Cynara scolymus were comparable to the predicted structures obtained from the respective Arabidopsis reference genes. The three dimentional models for GE578194.1, GE594969.1, GE583634.1 and GE599954.1 were predicted with $100 \%$ confidence by the Phyre2 software with a query coverage range from $88-99 \%$ in the structures. A comparison of deduced tertiary structures of both Cynara scolymus and Arabidopsis thaliana are given in (Fig 4a-1).

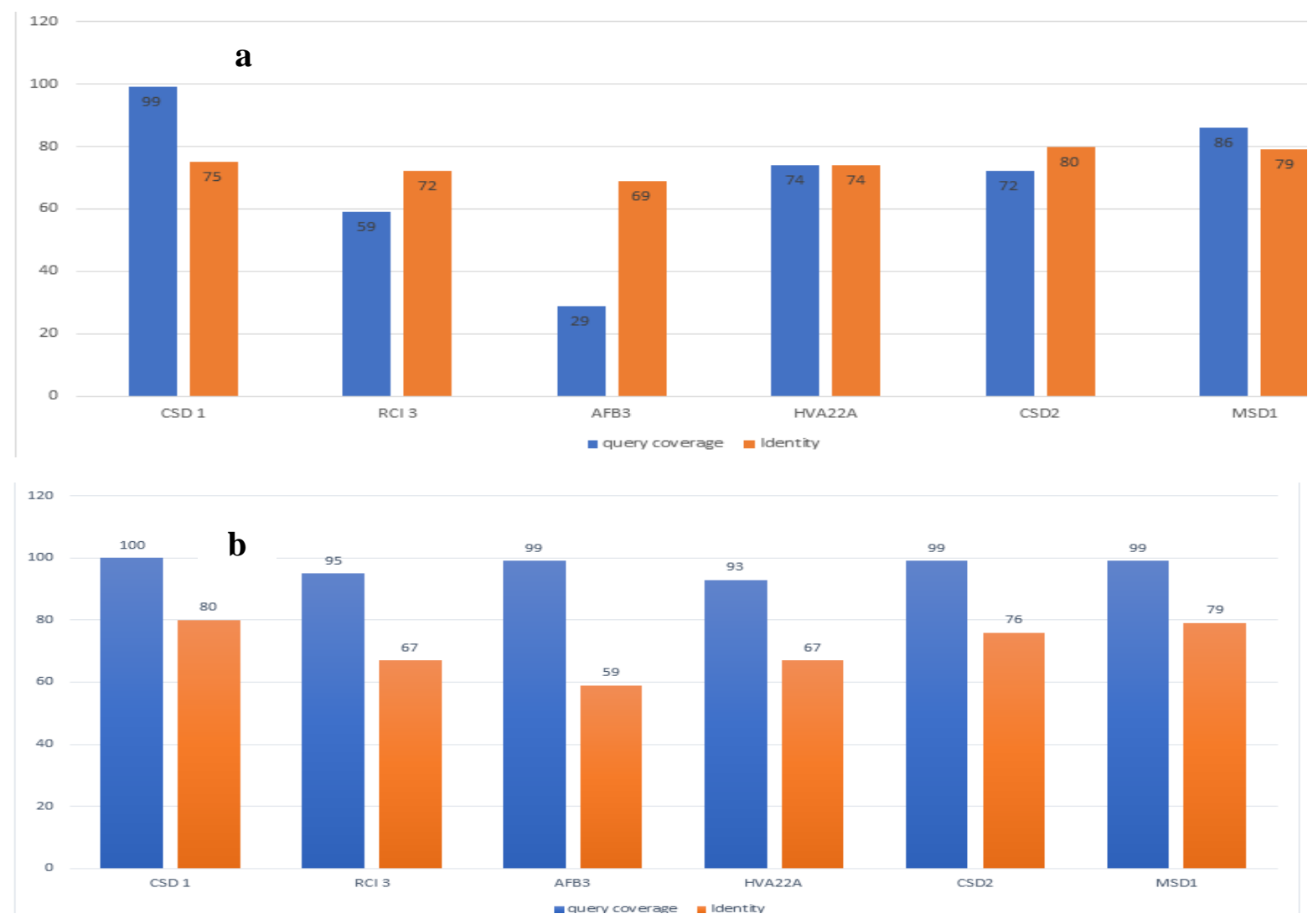

Figure 1(a). BLASTn results showing Nucleotide query coverage and identity of 6 salt stress genes in $C$. scolymus. (b) BLASTp results showing amino acids query coverage and identity of 6 salt stress genes in C. scolymus 


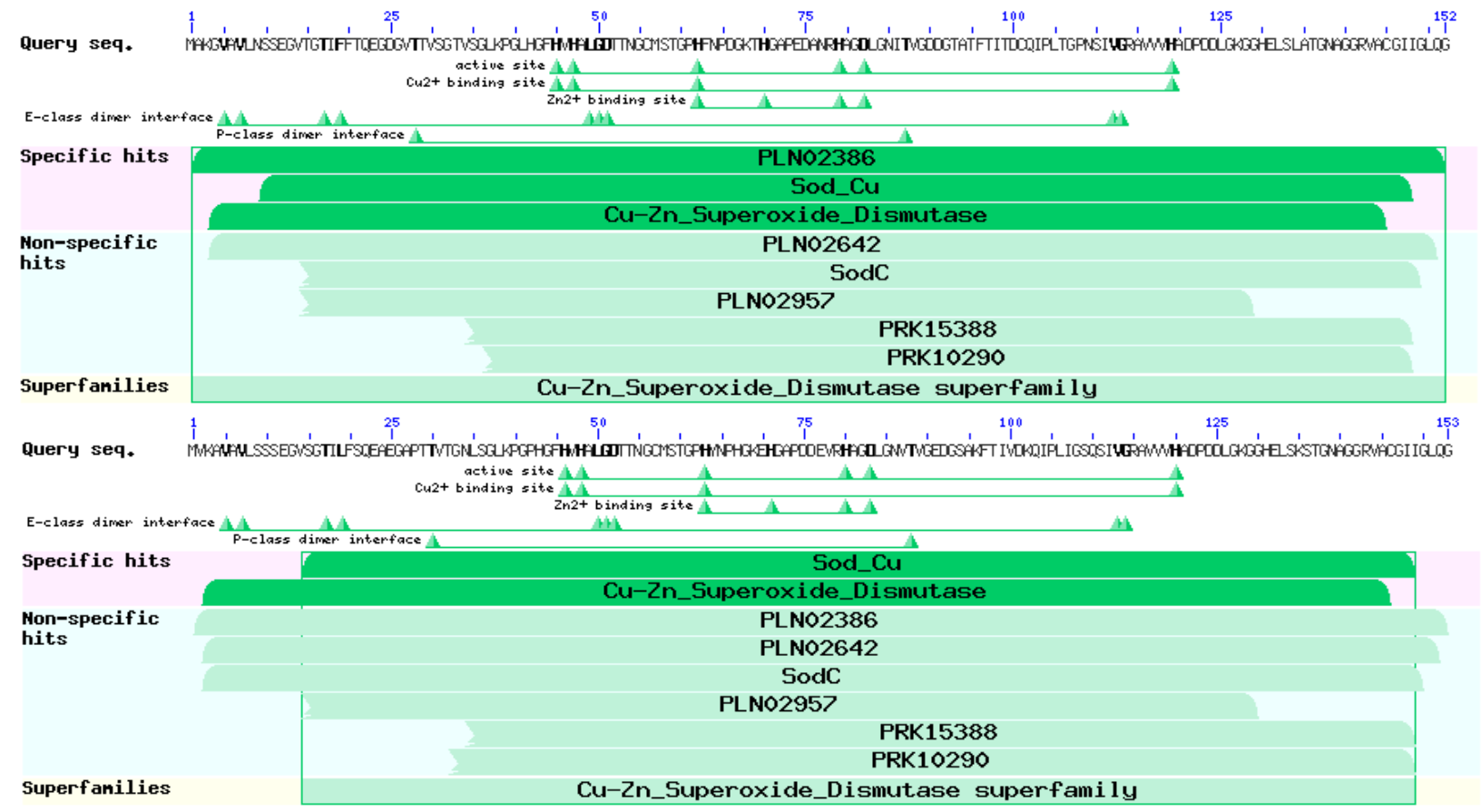

Figure 2a. Comparison of conserved domains found in CSD1 genes of Arabidopsis thaliana (above) and Cynara scolymus (below) showing presence of similar domains predicted by CD search

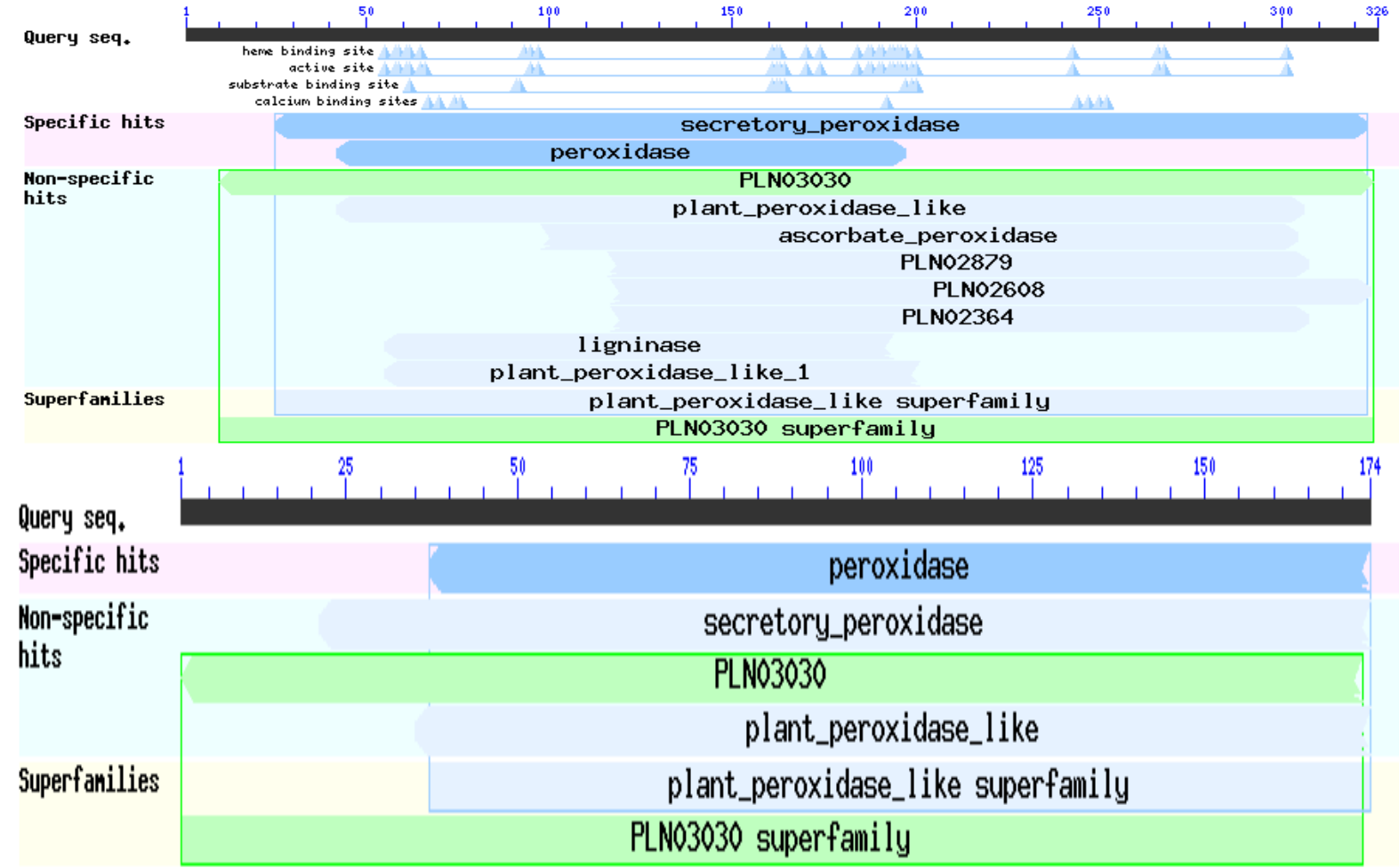

Figure 2b. Comparison of conserved domains found in RCI3 genes of Arabidopsis thaliana (above) and Cynara scolymus (below) showing presence of similar domains predicted by CD search 


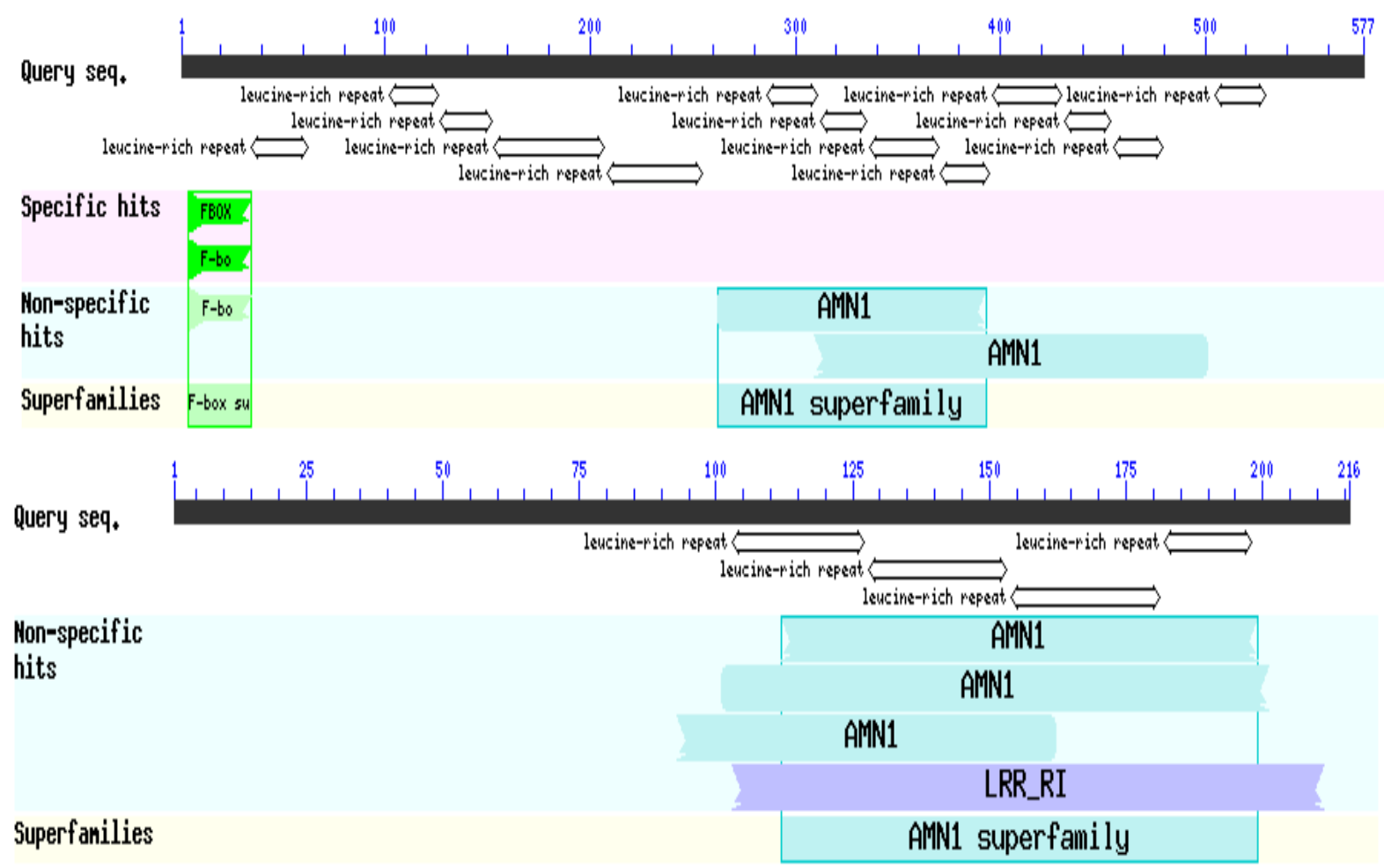

Figure 2c. Comparison of conserved domains found in AFB3 genes of Arabidopsis thaliana (above) and Cynara scolymus (below) showing presence of similar domains predicted by CD search

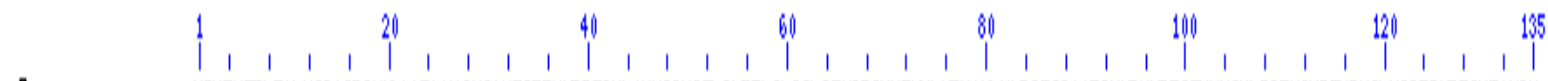

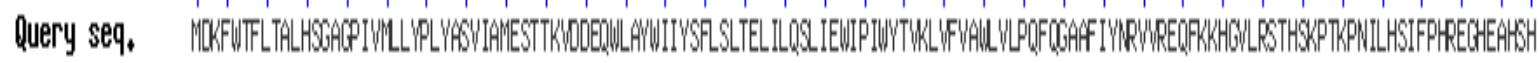

specific hits

Hon-specific

hits

superfatilies

TB2_DP1_HWA22

YOP1

TB2_LP1_HWA22 superfamily

Quer'y seq,

specific hits

Hon -specific

hils

Superfalilies

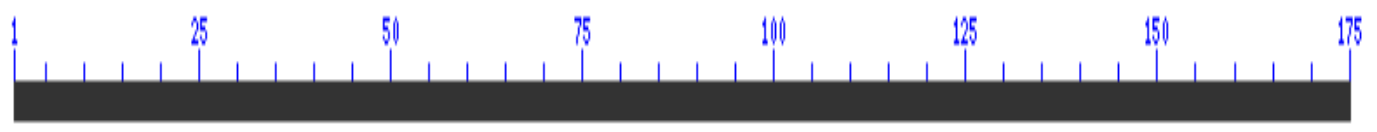

Figure 2d. Comparison of conserved domains found in HVA22A genes of Arabidopsis thaliana (above) and Cynara scolymus (below) showing presence of similar domains predicted by CD search 


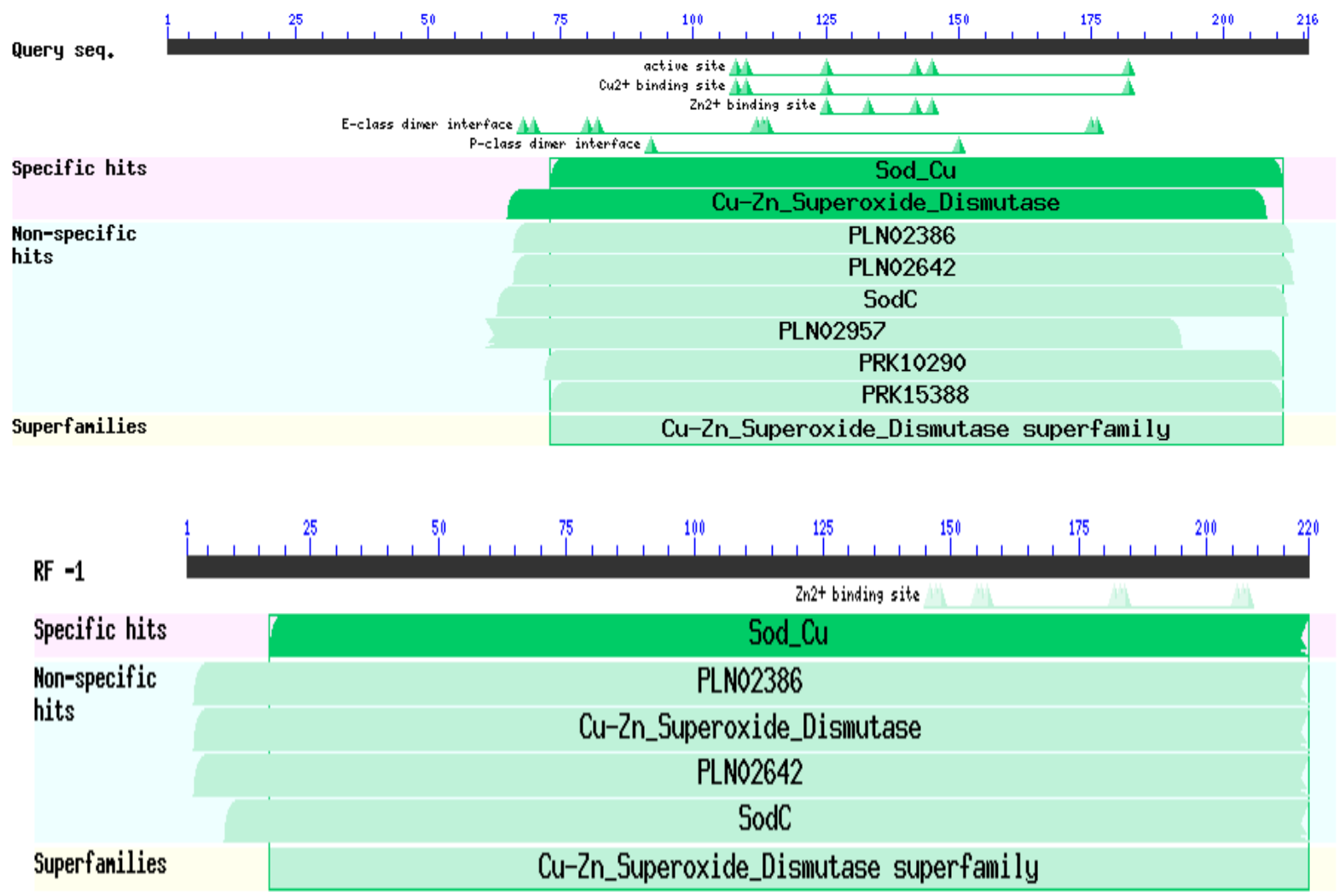

Figure 2e. Comparison of conserved domains found in CSD2 genes of Arabidopsis thaliana (above) and Cynara scolymus (below) showing presence of similar domains predicted by CD search

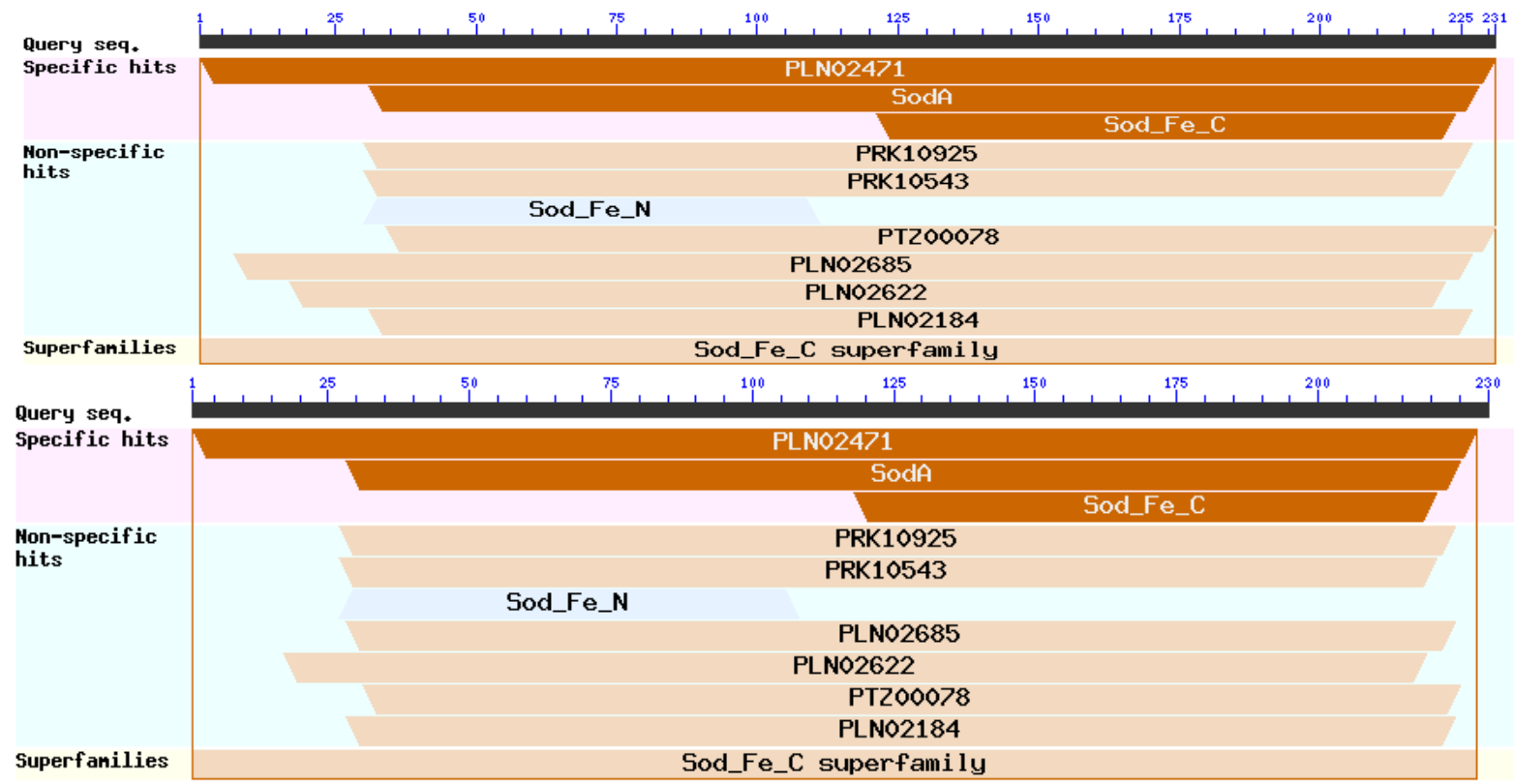

Figure 2f. Comparison of conserved domains found in MSD1 genes of Arabidopsis thaliana (above) and Cynara scolymus (below) showing presence of similar domains predicted by CD search 
$\mathbf{a}$

Arabidopsis thaliana

Camelina sativa

Papaver somniferum

Cynara scolymus

Arabidopsis thaliana

Camelina sativa

Papaver somniferum

Cvnara scolvmus

Arabidopsis thaliana

Camelina sativa

Papaver somniferum

Cynara scolymus

Arabidopsis thaliana

Camelina sativa

Papaver somniferum

Cynara scolymus
MAIRCVASRKTLAGLKETSSRLLRIRGIQTFTLPDLPYDYGALEPAISGEIMQIHHQKHH MAIRCVASRKTLAGLKETSSRLIGFRGIQTFTLPDLPYDYGALEPAISGEIMQIHHQKHH MALRTVMSRKSLGLAK --.-LGFSNARGLQTFSLPDLDYDYGALEPAISGEIMQIHHOKHH MALRTLLSGKTLGALS --RFQQQIRGLQTFTLPDLAYDYGALEPAISGEIMELHHQKHH $* * ; * * * ; *$, $* * ; * * * ; * * * ; * * * * * * * * * * * * * * ; ; * * * * * *$

QAYVTNYNNALEQLDQAVNKGDASTVVKLQSAIKFNGGGHVNHSIFINKNLAPSSEGGGEP QTYVTNYNNALEQLDQAVSKGGTSAVVKLQSAIKFNGGGHVNHSIFIJKNLAPVKEGGGEP QAYITNYNKALEQLTEATAKGDTSTVVKLQSAIKFNGGGHVNHSIFIKNLLTPVGQGGGEL QTYITNYNKALEQLDDAIAKGDASTAVKLQSAIKFNGGGHVNHSIFWKNLAPTHEGGGEP

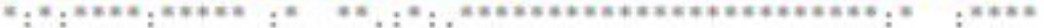

PKGSLGSAIDAHFGSLEGLVKKMSAEGAAVQGSGWVWLGLDKELKKLVVDTTANQDPLVT PKGSLGSAIDTHFGSLEGLVKKMSAEGAAVQGSGWVWLGLDKELKKLVVDTTANQDPLVT PRGSLASAIDTHFGSFEKLVQKANAEGAALQGSGWVWLGLDKELKKLVVETTANQDPLVT PHGSLGNAIDQHFGSMEKLIAKMNTEGAAVQGSGIVWLAVDKELKRLVIETTANQDPLVT

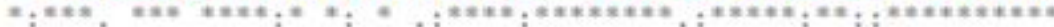

KGGSLVPLVGIDVWEHAYYLQYKINVRPEYLKNVWKVINWKYASEVYEKENN-- 231 KGGSLVPLVGIDVWEHAYYLQYKNVRPEYLKNVWKVINWKYASEIYEKECN - - 231 KGANLVPLLGIDVWEHAYYLQYKNVRPDYLKNIWKVINWKYASEVYEK …. 225 KGASLVPLIGIDVWEHAYYQYKINVRDYLKNIWKVINWKYASEVYEKECPFK 230 $* *, * * * * ; * * * * * * * * * * * * * * * * * * ; * * * * ; * * * * * * * * * * * ; * * *$

b

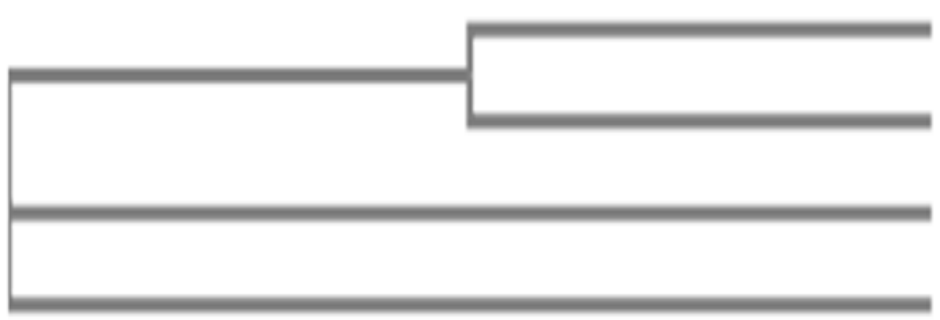
Arabidopsis thaliana
Camelina sativa
Papaver somniferum
Cynara scolymus

Figure 3(a). Alignment of Cynara scolymus MSD1 gene sequence with Arabidopsis thaliana, Camelina sativa and Papaver somniferum. Below the protein sequences is a key denoting conserved sequence (*), conservative mutations (:), semi-conservative mutations (.), and nonconservative mutations ( ). Alignment was generated by Clustal $O$ (1.2.4).

(b) Cladogram of Cynara scolymus MSD1 gene sequence with Arabidopsis thaliana, Camelina sativa and Papaver somniferum showing that on the basis of MSD1 gene sequence, Cynara scolymus is more close to Papaver somniferum as compared to Arabidopsis thaliana and Camelina sativa

Table 2. Percent Identity Matrix of MSD1 gene predicted in Cynara scolymus by comparison with that of Camelina sativa, Papaver somniferum and Arabidopsis thaliana. Closeness of Cynara scolymus with Papaver somniferum is highlighted in dark blue colour

\begin{tabular}{|l|c|c|c|c|c|}
\hline 1 & Arabidopsis thaliana & 100.00 & 94.37 & 80.89 & 79.82 \\
\hline 2 & Camelina sativa & 94.37 & 100.00 & 80.44 & 78.51 \\
\hline 3 & Papaver somniferum & 80.89 & 80.44 & 100.00 & 80.44 \\
\hline 4 & Cynara scolymus & 79.82 & 78.51 & 80.44 & 100.00 \\
\hline
\end{tabular}


Table 3. Comparison of molecular structures of deduced proteins from Arabidopsis thaliana reference genes and Cynara scolymus target ESTs obtained by ProtParam

\begin{tabular}{|c|c|c|c|c|c|c|c|c|c|c|c|c|}
\hline \multirow[b]{2}{*}{ Parameters } & \multicolumn{6}{|c|}{ Arabidopsis thaliana Reference Genes } & \multicolumn{6}{|c|}{ Cynara scolymus Target ESTs } \\
\hline & 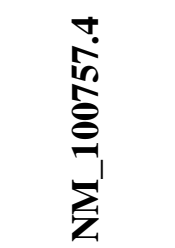 & 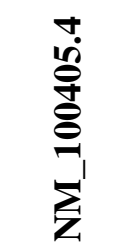 & 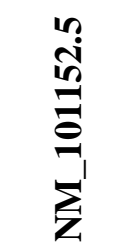 & 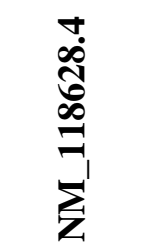 & 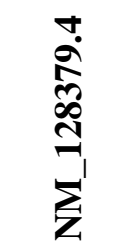 & 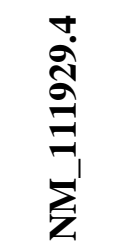 & 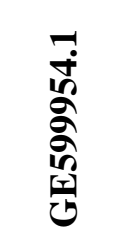 & $\frac{7}{\dot{5}}$ & 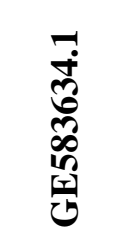 & 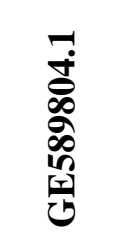 & 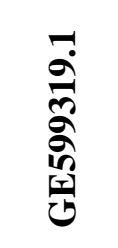 & 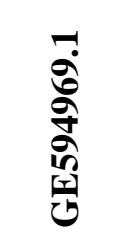 \\
\hline $\begin{array}{c}\text { Gene Symbol/BlastP } \\
\text { Result }\end{array}$ & CSD1 & RCI3 & AFB3 & $\begin{array}{c}\text { HVA22 } \\
\text { A }\end{array}$ & CSD2 & MSD1 & & & & & & \\
\hline Stop ORF & 611 & 1041 & 2115 & 612 & 787 & 771 & 495 & 736 & 851 & 623 & 283 & 724 \\
\hline ORF length & 459 & 981 & 1734 & 408 & 651 & 696 & 462 & 525 & 648 & 528 & 219 & 693 \\
\hline \# of $\mathbf{A A}$ & 152 & 326 & 577 & 135 & 216 & 231 & 153 & 174 & 216 & 175 & 72 & 230 \\
\hline Mol. Wt & 15097.68 & $\begin{array}{c}34905 . \\
70\end{array}$ & $\begin{array}{c}64906 . \\
18\end{array}$ & $\begin{array}{c}15753.4 \\
1\end{array}$ & $\begin{array}{c}22243 . \\
95\end{array}$ & $\begin{array}{c}25443 . \\
94\end{array}$ & $\begin{array}{c}15351 . \\
05\end{array}$ & $\begin{array}{c}18532.5 \\
4\end{array}$ & $\begin{array}{c}24712 . \\
52\end{array}$ & $\begin{array}{c}20068 . \\
94\end{array}$ & $\begin{array}{c}8250 . \\
63\end{array}$ & $\begin{array}{l}25729 . \\
34\end{array}$ \\
\hline Instability index & 14.73 & 36.16 & 45.62 & 31.36 & 27.46 & 29.27 & 21.49 & 48.88 & 54.79 & 39.79 & 24.24 & 33.97 \\
\hline Alpha helix (\%) & 0 & 48 & 36 & 92 & 4 & 60 & 0 & 59 & 39 & 87 & 49 & 58 \\
\hline Beta strand (\%) & 39 & 0 & 12 & 0 & 38 & 10 & 43 & 0 & 8 & 0 & 22 & 9 \\
\hline TM helix (\%) & - & 5 & - & 45 & - & - & - & 9 & - & 39 & - & - \\
\hline \multicolumn{13}{|c|}{ Tertiary Structure } \\
\hline Residues & 152 & 301 & 569 & 12 & 154 & 202 & 152 & 153 & 210 & 56 & 47 & 202 \\
\hline Confidence of Model & 100 & 100 & 100 & 30 & 100 & 100 & 100 & 100 & 100 & 51 & 44.4 & 100 \\
\hline Coverage & 100 & 92 & 99 & 9 & 71 & 87 & 99 & 88 & 97 & 32 & 65 & 88 \\
\hline \multicolumn{5}{|c|}{ Arabidopsis reference genes tertiary structure analogues } & \multicolumn{8}{|c|}{ Cynara scolymus EST tertiary structure analogues } \\
\hline
\end{tabular}




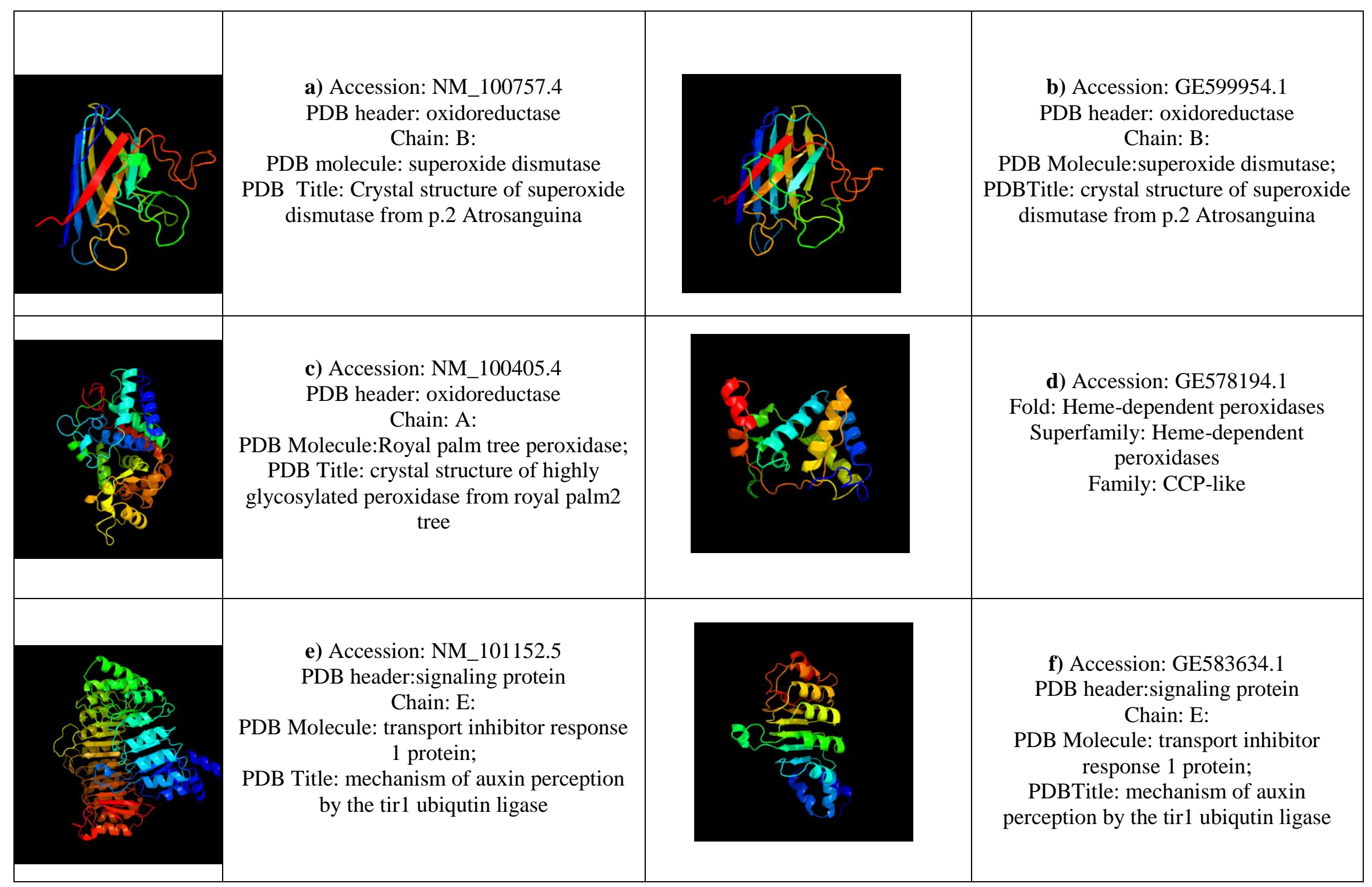




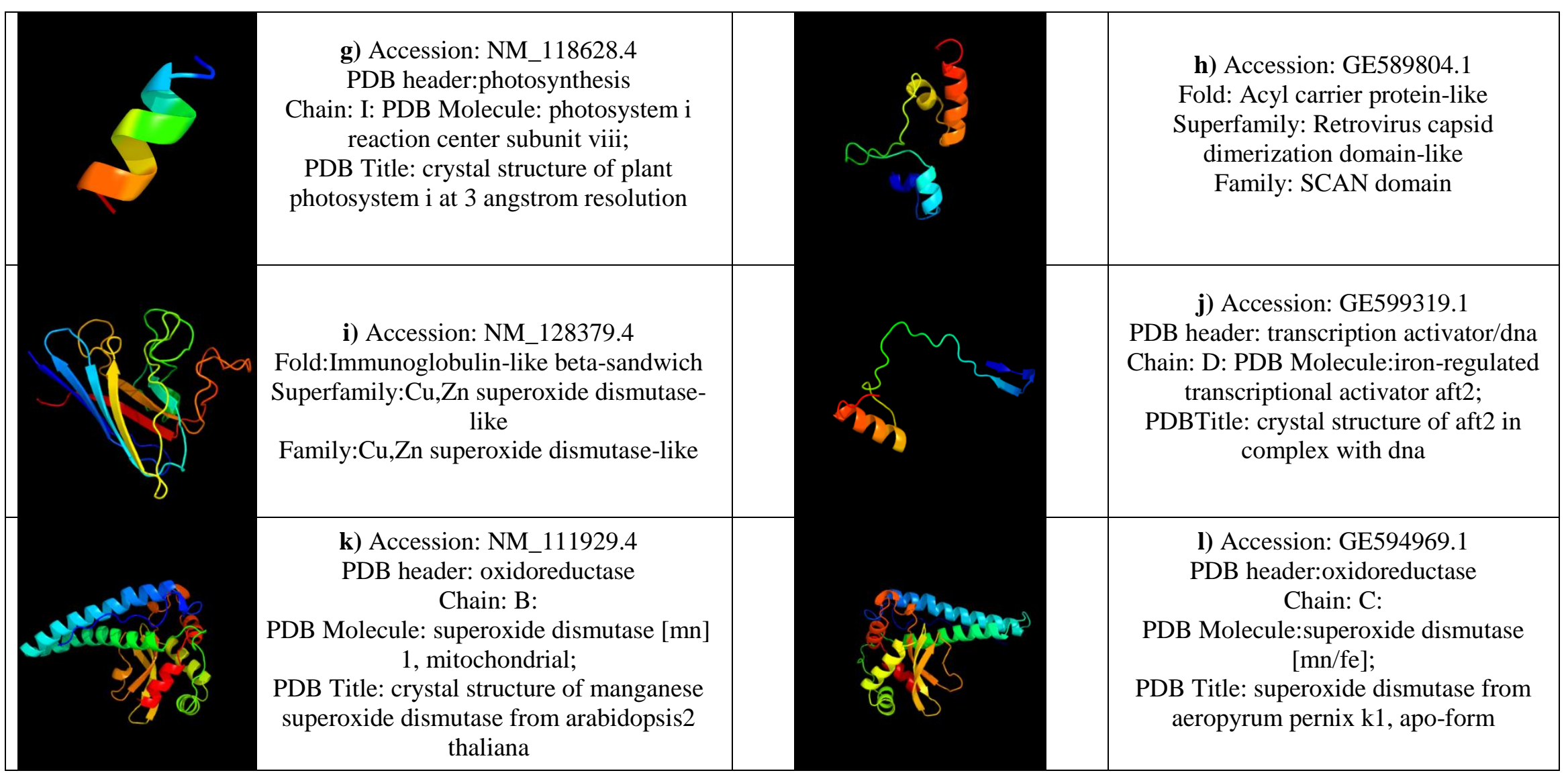

Fig 4 (a-l). Tertiary structure analogues obtained by Phyre2 online analysis server from deduced proteins of Arabidopsis thaliana reference genes and Cynara scolymus target ESTs 


\section{Discussion}

Cynara scolymus L. thrives well in saline areas and is regarded as a moderately salt resistant crop, but very little information is available about its salt stress tolerance mechanism. However various researchers have worked out to comprehend the effect of salt stress on phenolic compounds and antioxidant activity of its leaves [8]. The microRNA (miRNA) analysis for Cynara scolymus has also been carried and many salt stress genes are reported to be targeted by miRNAs [15].

This study resulted in the bioinformatics identification of salt stress genes in this important plant. All selected genes have been predicted in ESTs; confirming their expression in Cynara scolymus. Results completely supported our hypothesis.

Many researchers have used bioinformatics approach to identify salt stress responsive genes and microRNAs in plants $[16,17]$. EST analysis is an important approach to identify new conserved genes [18]. Researchers prefer to screen out the EST sequences (if available) to find out conserved sequences due to confirmation of their expression [19, 20]. To understand the transcriptional profiling of genes whose expression levels alter in response to salt stress, different EST/cDNA collections have been utilized in plants [21].

As compared to nucleotide sequences query coverage, protein sequence query coverage is greater due to codon degeneracy and due to the fact that DNA alignment doesn't take into account the redundancy of amino acid codons. Furthermore, DNA alignment also doesn't consider the more similar structures of some amino acids to others and the similar functional role they have in the protein e.g. Isoleucine and valine have similar structures, both have hydrophobic side chains and differ only by the addition of an extra carbon on isoleucine. A mutation from one to the other is not as likely to substantially change the protein structure as some other mutations would, but a DNA sequence alignment treats this mutation the same as any other. Therefore both approaches were adopted in this research. The higher query coverage in case of BlastP as compared to Blastn for all six genes is upto the mark.

This research also investigated the presence of conserved domains on reference genes (Arabidopsis thaliana) and their predicted orthologs (Cynara scolymus L.) Presence of same conserved domains in all predicted genes provides a clue of similar function. All the predicted genes share similar super families and specific hits and therefore a high confidence level for the inferred function of the protein query sequence.

The amino acid sequence of a protein is known to determine the secondary and tertiary structure of a protein molecule [22]. In the current analysis the deduced secondary protein structures also show comparable physical properties including molecular stability, aliphatic index and GRAVY thus infering similar results as above. This is also evident by the presence of trans-membrane helices in Arabidopsis reference genes $\mathrm{RCl} 3$ and HVA22A and their target ESTs in Cynara scolymus (Accession No.s GE578194.1 and GE589804.1). It is known that genes for proteins with structural and functional similarity have similarities in secondary structures leading to comparable three dimentional tertiary structures. This is relevant for similarity in their functional properties. Moreover, the tertiary structure analogs of Cynara scolymus EST Acession GE599954.1 (superoxide dismutase), GE578194.1 (heme-dependent peroxidase), GE583634.1 (transport inhibitor response 1 protein) and GE594969.1 (molecule superoxide dismutase) showed 100\% confidence of model with analogs of their respective Arabidopsis reference genes. These evident similarities lead to the conclusion that the targeted ESTs possessed 
similar structure and function as their reference genes. Thus, indicating the possibility of their orthologous origin [23]. Many scientists are pursuing similar in-silico (bioinformatics) approaches to identify genes related to stress tolerance in plants from available gene pool of plant genomes [24, 25]. This saves researchers from unwanted waste of time and potential funds. Due to the above findings it is plausible to say that Arabidopsis conserved domains can be used to predict orthologs in Cynara scolymus which can further be used to identify potential genes for salt tolerance in plants.

\section{Conclusion}

It is predicted that in C. scolymus CSD1, MSD1, CSD2, AFB3, RCI3 and HVA22A salt stress responsive genes are present which may potentially help this plant against salinity. Bioinformatics identification of salt stress responsive genes in C. scolymus and their molecular annotation will be a good contribution in understanding the acclimation process of this plant under saline conditions.

\section{Authors' contributions}

Conceived and designed the experiments

IA Baloch \& S Johar,

Performed the experiments:

S Johar, BH Mengal \& AK Alvi,

Analyzed the data: MN Shawani \& SS

Qambrani,

Wrote the paper:

IA Baloch \& B Sadia.

\section{References}

1. Llorach R, Espin JC, Tomas-Barberan FA \& Ferreres F (2002). Artichoke (Cynara scolymus L.) byproducts as a potential source of health promoting antioxidant phenolics. $J$ Agr Food Chem 50(12): 3458-3464.

2. Fratianni F, Tucci M, De Palma M, Pepe R \& Nazzaro F (2007). Polyphenolic composition in different parts of some cultivars of globe artichoke (Cynara cardunculus L. var. Scolymus (L.) Fiori). Food Chem 104(3): 1282-1286.
3. Ceccarelli N, Curadi M, Picciarelli P, Martelloni L, Sbrana C \& Giovannetti M (2010). Globe artichoke as a functional food. Mediterranean J. Nutrition and Metabolism 3(3): 197-201.

4. Lattanzio V, Kroon PA, Linsalata V \& Cardinali A (2009). Globe artichoke: a functional food and source of nutraceutical ingredients. J Funct Foods 1(2): 131-144.

5. Pandino G, Lombardo S, Mauromicale G \& Williamson G (2011). Phenolic acids and flavonoids in leaf and floral stem of cultivated and wild Cynara cardunculus L. genotypes. Food Chem 126(2): 417422.

6. Breyne P \& Zabeau M (2001). Genomewide expression analysis of plant cell cycle modulated genes. Curr Opin Plant Biol 4(2): 136-142.

7. Benlloch-González M, Fournier JM, Ramos J \& Benlloch M (2005). Strategies underlying salt tolerance in halophytes are present in Cynara cardunculus. Plant Sci 168(3): 653-659.

8. Rezazadeh A, Ghasemnezhad A, Barani M \& Telmadarrehei T (2012). Effect of salinity on phenolic composition and antioxidant activity of artichoke (Cynara scolymus L.) leaves. Res J Med Plant 6: 245-252.

9. Brands A \& Ho THD (2002). Function of a plant stress-induced gene, HVA22. Synthetic enhancement screen with its yeast homolog reveals its role in vesicular traffic. Plant Physiol 130(3): 1121-1131.

10. Attia H, Arnaud N, Karray N \& Lachaâl M (2008). Long-term effects of mild salt stress on growth, ion accumulation and superoxide dismutase expression of Arabidopsis rosette leaves. Physiol Plant 132(3): 293-305.

11. Jalali-e-Emam SMS, Alizadeh B, Zaefizadeh M, Zakarya RA \& Khayatnezhad M (2011). Superoxide 
dismutase (SOD) activity in $\mathrm{NaCl}$ stress in salt-sensitive and salt-tolerance genotypes of Colza (Brassica napus L.). Middle East J Sci Res 7: 7-11.

12. Gong Y, L Rao \& D Yu. (2013). Abiotic stress in plants. Agricultural chemistry. Inter Tech Rijeka 113-152.

13. Gasteiger E, Hoogland C, Gattiker A, Duvaud S, Wilkins MR, Appel RD \& Bairoch A (2005). Protein Identification and Analysis Tools on the ExPASy Server; (In) John M. Walker (ed): The Proteomics Protocols Handbook, Humana Press, pp 571-607.

14. Kelley LA, Mezulis S, Yates CM, Wass MN \& Sternberg MJ (2015). The Phyre2 web portal for protein modeling, prediction and analysis. Nat Protoc 10(6): 845.

15. De Paola D, Cattonaro F, Pignone D \& Sonnante G (2012). The miRNAome of globe artichoke: conserved and novel micro RNAs and target analysis. $B M C$ Genomics 13(1): 41.

16. Sunkar R, Chinnusamy V, Zhu J \& Zhu JK (2007). Small RNAs as big players in plant abiotic stress responses and nutrient deprivation. Trends Plant Sci 12(7): 301-309.

17. Jagadeesh Selvam HRN, Senthil N, Manikanda Boopathi N \& Raveendran M (2015). Computational identification of salinity responsive microRNAs in contrasting genotypes of Finger millet (Eleusine coracana L.). Res J Biotechnol 10: 9 .

18. Yang RZ, Park S, Reagan WJ, Goldstein R, Zhong S, Lawton M, ... \& Gong DW (2009). Alanine aminotransferase isoenzymes: molecular cloning and quantitative analysis of tissue expression in rats and serum elevation in liver toxicity. Hepatol 49(2): 598-607.
19. Baloch IA, Barozai MYK \& Din M (2015). Identification and characterization of 25 microRNAs and their targeted proteins in apricot (Prunus armeniaca L.). JAPS: J Anim Plant Sci 25(5).

20. Baloch IA, Barozai MYK \& Din M (2018). Bioinformatics prediction and annotation of cherry (Prunus avium L.) microRNAs and their targeted proteins. Turkish J Bot 42(4): 382-399.

21. Shiozaki N, Yamada M \& Yoshiba Y (2005). Analysis of salt-stress-inducible ESTs isolated by PCR-subtraction in salt-tolerant rice. Theor Appl Genet 110(7): 1177-1186.

22. Marks DS, Hopf TA \& Sander C (2012). Protein structure prediction from sequence variation. Nat Biotechnol 30(11):1072.

23. Altenhoff AM, Studer RA, RobinsonRechavi M \& Dessimoz C (2012). Resolving the ortholog conjecture: orthologs tend to be weakly, but significantly, more similar in function than paralogs. PLoS Comput Biol 8(5): p.e1002514.

24. Sheoran S, Pandey B, Sharma P, Narwal S, Singh R, Sharma I \& Chatrath R (2013). In silico comparative analysis and expression profile of antioxidant proteins in plants. Genet Mol Res 12(1): 537-551.

25. Ozyigit II, Filiz E, Vatansever R, Kurtoglu KY, Koc I, Öztürk MX \& Anjum NA (2016). Identification and comparative analysis of $\mathrm{H} 2 \mathrm{O} 2-$ scavenging enzymes (ascorbate peroxidase and glutathione peroxidase) in selected plants employing bioinformatics approaches. Front Plant Sci 7: 301. 